\section{MS39-P18 DIALS - a new toolbox for diffraction image integration}

David G. Waterman ${ }^{1,2}$, Graeme Winter ${ }^{3}$, James M. Parkhurst ${ }^{3}$, Luis Fuentes-Montero ${ }^{3}$, Richard J. Gildea ${ }^{3}$, Aaron S. Brewster ${ }^{4}$, Nicholas K. Sauter ${ }^{4}$, Gwyndaf Evans ${ }^{3}$

1. $\mathrm{CCP} 4, \mathrm{STFC}$

2. RCaH, Rutherford Appleton Laboratory

3. Diamond Light Source

4. Lawrence Berkeley National Laboratory

email: david.waterman@stfc.ac.uk

DIALS is a collaborative initiative to produce an open source software toolbox, built on top of the cctbx, to address tasks in diffraction image integration. The toolbox has been developed with a flexible, modular design and can be used at various levels: as a library, via a comprehensive set of command-line tools, or driven by a high-level script or expert system such as xia2. The motivation behind the development of DIALS is discussed and the structure of the software is explained. In particular, the use of DIALS for fast, high quality integration by $3 \mathrm{D}$ profile fitting in the context of synchrotron rotation experiments is covered in detail. Key features of DIALS are explored, including a powerful indexing program that utilises a choice of methods for identifying candidate basis vectors, and global multi-experiment geometry refinement with smoothly varying crystal models. The use of DIALS in different experimental contexts such as serial snapshot crystallography is touched upon. Potential users are encouraged to get involved with the project, and a timeline of development plans culminating in beta release of the software in the CCP4 suite is presented.

Keywords: integration, software, cctbx, ccp4, open source

\section{MS39-P19 High resolution powder X-ray diffraction beamline at Taiwan Photon Source}

Hwo-Shuenn Sheu ${ }^{1}$, Yu-Chun Chuang ${ }^{1}$, Chung-Kai Chang ${ }^{1}$, Kuan-Li Yu ${ }^{1}$, Longlife Lee ${ }^{1}$

\section{National Synchrotron Radiation Research Center, Taiwan}

email: hsheu@nsrrc.org.tw

The low emittance (1.6 nm-rad) synchrotron radiation ring, Taiwan Photon Source (TPS), has reached the design goal of $3 \mathrm{GeV}$ electron beam stored and delivered its first synchrotron light on the last day of 2014. The first $1.5 \mathrm{GeV}$ synchrotron radiation ring in Taiwan, Taiwan Light source (TLS), has been operated for 21 years. TLS serves more than 2000 scientists and students every year now. To response the highly demanding of hard X-ray users, the medium energy $3 \mathrm{GeV}$ synchrotron ring, TPS, has been constructed since 2010. In phase I, seven frontier beamlines will be constructed by the end of 2015 . A dedicated high resolution powder diffraction beamline will be built after then. A highly collimated and intense $\mathrm{X}$-ray source will be produced by an in-vacuum undulator (IU22) to obtain the highest possible brilliance in the range of 5-30 keV. To meet the versatile researchers in chemistry, physics and materials, a large concentric 3-circle diffractometer (Newport) equipped with a multi-crystal analyzer system and a fast position sensitive detector (MYTHEN 24K) were designed for high angular resolution and time-resolved studies respectively. The polycrystalline materials under non-ambient conditions, such as high/low temperature, high pressure and gas de/adsorption, will be investigated by PXRD techniques. In addition, to satisfy the high demanding of powder users, large amount of samples and increase the beamtime efficiency, a high throughput robot will be installed to allow automated sample mounting. The in situ and time resolved experiments as well as structure determination from powder diffraction data will be emphasized in this beamline.

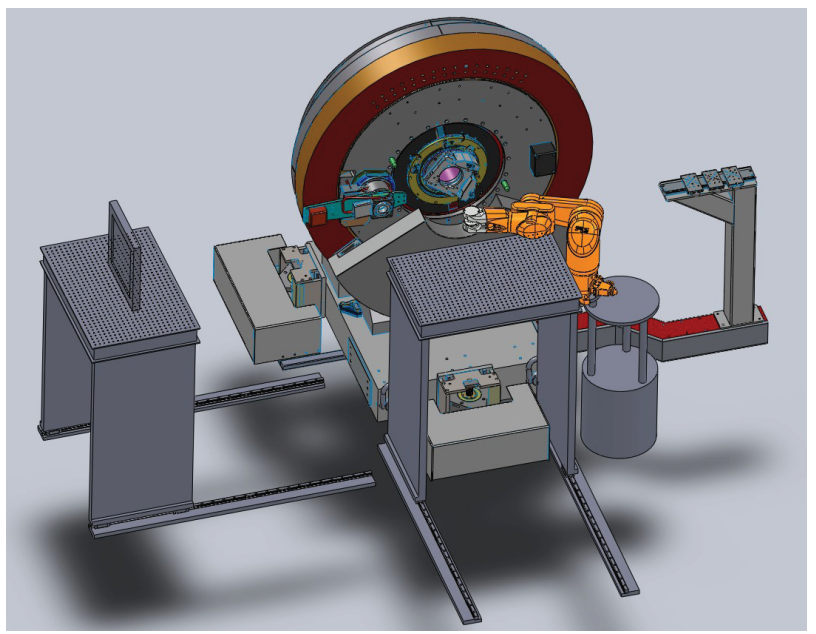

Figure 1. A large three circle powder diffractometer equiped with a multi-crystal analyzer, strip detector and flat area detector will be installed in TPS.

Keywords: Synchrotron Radiation, Powder X-ray Diffraction 